\title{
Хорошо определенные логики ${ }^{1}$
}

\author{
И. А. ГорБУнов
}

\begin{abstract}
Some questions concerned the deduction theorem for consequence operations and sentential calculi are considered in the present paper.

Ключевые слова: теорема о дедукции, дедуктивные пропозициональные системы
\end{abstract}

\section{1 Предисловие}

В отношении ее математического содержания данная работа является скорректированным и доработанным пересказом некоторых фактов из работы Ризарда Вуйцицкого [1] (или см. [2]). В частности утверждения 6-10 и 12 являются несколько уточненными фактами из указанной работы. К сожалению, в оригинале эти факты сформулированы и доказаны не совсем корректно. Утверждения 11, 13 и 14 доказаны и сформулированы автором этой работы.

\section{2 Введение}

Мы пока не будем определять, что такое логика, а будем опираться на ее интуитивное понимание. Отметим только, что всякая логика определяет (и в синтаксическом случае сама полностью определяется через) отношение логического следования, устанавливаемое между списками предложений языка и предложениями языка. Это отношение сопоставляет списку предложений, называемых посылками, некоторое предложение, которое называется их следствием.

Отношение логического следования, в свою очередь, некоторым образом выражается в естественном языке. Таким образом,

\footnotetext{
${ }^{1}$ Работа выполнена при поддержке РФФИ, гранты 08-06-00414 и 10-06-00360.
} 
выделение логики языка посредством этого же языка предполагает, что язык уже располагает некоторыми средствами представления логического следования. То есть в нем существует логическая связка (или формула), назначение которой - выражать в пропозициональном языке отношение логического следования. Если в языке существует такая связка, то, видимо, ее и стоит называть импликащией. Связку эту, как обычно, будем обозначать $\rightarrow$.

Частным примером импликации является материальная импликация. Обычно в русском языке эта связка передается включением в предложение словосочетания «Если ..., то...».

Изложение всякой логики происходит в русском же языке и в таком изложении мы вполне можем ожидать появления предложения следующего вида: «Если мы имеем формулу $\varphi$ в качестве посылки, то имеем формулу $\psi$ в качестве следствия». В этом предложении импликация представляет отношение выводимости (которое обычно обозначается символом $\vdash$ ), существующее в логике. Совокупность всех таких предложений языка, видимо, имеет свою логику. В этой работе мы попробуем приблизиться к этой логике и исследуем, какими же свойствами должны обладать логики, в которых импликация представляет отношение выводимости.

Рассмотрим следующую модель пропозиционального языка. Пусть $V$ - счетное множество символов, называемых пропозиииональными переменными, и $\Sigma$ - не более чем счетное множество конечноместных функциональных символов, называемых пропозиииональными связками. Пару $\langle V, \Sigma\rangle$ будем называть пропозичиональным алфавитом. Всякий терм, построенный из символов алфавита $\langle V, \Sigma\rangle$, будем называть формулой. Языком $S$ будем называть множество всех формул алфавита $\langle V, \Sigma\rangle$. Подстановку определим обычным образом, т. е. как гомоморфизм $\varepsilon: S \rightarrow S$, который является продолжением отображения $\varepsilon: V \rightarrow S$. Обозначим через $\mathbf{E}$ множество всех подстановок.

Функцию $C: 2^{S} \rightarrow 2^{S}$ будем называть операцией $n р и с о е д и$ нения следствий над языком $S$, или, кратко, следованием, если для любых $X, Y \in 2^{S}$

A1. $X \subseteq C(X)$, 
A2. $C(X)=C(C(X))$,

A3. $X \subseteq Y \Rightarrow C(X) \subseteq C(Y)$,

A4. множество $C(\varnothing)$ замкнуто относительно подстановки.

Следование $C$ будем называть структурным, если для любой подстановки $\varepsilon$ и любого множества формул $X$ выполняется условие $\varepsilon(C(X)) \subseteq C(\varepsilon(X))$. Следование будем называть финитарным, если для любого $X$ верно, что $C(X)=\bigcup_{Y \subseteq X} C(Y)$, где $Y$ - конечное множество. Структурное и финитарное следование будем называть стандартным.

Пару $\langle S, C\rangle$, где $C$ - структурная операция присоединения следствий, будем называть пропозищиональной логикой, или, для краткости, логикой.

Любое подмножество $\rho \subseteq 2^{S} \times S$ будем называть правилом, а любой элемент этого подмножества будем называть схемой. Будем говорить, что правило $\rho$ порождено схемой $\langle X, \alpha\rangle$, если $\rho=\{\langle\varepsilon(X), \varepsilon(\alpha)\rangle \mid \varepsilon \in \mathbf{E}\}$. Такое правило будем обозначать $\rho_{X / \alpha}$ (или, если это не будет вызывать недоразумений, просто $X / \alpha)$ и называть секвениией. Секвенцию $\varnothing / \alpha$ будем называть тавтологией и обозначать ее просто $\alpha$.

Множество $X \in 2^{S}$ будем называть замкнутым относительно правила $\rho$, если для любого $Y \subseteq X$ и для любого $\alpha \in S$ верно, что если $\langle Y, \alpha\rangle \in \rho$, то и $\alpha \in X$. Будем говорить, что следование $C$ базируется на множестве правил въвода $R$ (символически обозначать $C=C_{R}$ ), если для любого $X \in 2^{S}$ множество $C(X)$ является наименьшим множеством, содержащим $X$ и замкнутым относительно каждого правила из $R$. Для данного следования $C$ множество $C(X)$ будем называть теорией со множеством аксиом $X$.

\section{3 Некоторые свойства операции присоединения следствий}

Множество следствий конечного множества $\left\{\alpha_{1}, \ldots, \alpha_{n}\right\}$ будем обозначать $C\left(\alpha_{1}, \ldots, \alpha_{n}\right)$. Множество следствий из множества $\{\alpha\} \cup X$ будем обозначать как $C(\alpha, X)$. Докажем некоторые свойства операции присоединения следствий.

TEOPEMA 1. Если $\alpha \in C(X)$, mo $C(\alpha, X)=C(X)$. 
Доказательство. Так как $X \subseteq\{\alpha\} \cup X$, то в силу свойства $A 3$ $C(X) \subseteq C(\alpha, X)$. Докажем обратное включение.

Заметим, что $\{\alpha\} \cup X \subseteq\{\alpha\} \cup C(X)=C(X)$ и значит верно, что $C(\alpha, X) \subseteq C(\{\alpha\} \cup C(X))=C(C(X))=C(X) \quad$ Q.E.D.

TEOPEMA 2. $Y \subseteq C(X) \Leftrightarrow C(Y) \subseteq C(X)$

Доказательство. $(\Rightarrow)$ Пусть $Y \subseteq C(X)$, тогда, в силу $A 2$ и $A 3$ верно, что $C(Y) \subseteq C(C(X))=C(X)$.

$(\Leftarrow)$ Пусть $C(Y) \subseteq C(X)$. В силу $A 1$ верно, что $Y \subseteq C(Y)$, тогда $Y \subseteq C(X)$.

Q.E.D.

СЛЕДСТВИЕ 1. $\alpha \in C(X) \Leftrightarrow C(\alpha) \subseteq C(X)$

TEOPEMA 3. $C(X) \cup C(Y) \subseteq C(X \cup Y)$

Доказательство. Так как $X \subseteq X \cup Y$ и $Y \subseteq X \cup Y$. Следовательно $C(X) \subseteq C(X \cup Y)$ и $C(Y) \subseteq C(X \cup Y)$. Таким образом $C(X) \cup C(Y) \subseteq C(X \cup Y)$.

Q.E.D.

TEOPEMA 4. $C(C(X) \cup C(Y))=C(X \cup Y)$

Доказательство. Включение $C(C(X) \cup C(Y)) \subseteq C(X \cup Y)$ следует из предыдущей теоремы. Докажем обратное включение. Так как $X \subseteq C(X)$ и $Y \subseteq C(Y)$, то $X \cup Y \subseteq C(X) \cup C(Y)$.

Q.E.D.

\section{4 Хорошо определенная логика}

Поскольку импликация в языке нашей логики должна выражать отношение выводимости из списка посылок, то язык должен содержать также связку, объединяющую посылки в список. Таким образом, будем рассматривать логики в языке, содержащем связки импликации $\rightarrow$ и конъюнкции $\wedge$. При этом предполагаем, что конъюнкция обладает свойствами коммутативности и ассоциативности. Поэтому посредством квазиформулы $\alpha_{1} \wedge \ldots \wedge \alpha_{n}$ будем обозначать конъюнкцию формул $\alpha_{1}, \ldots, \alpha_{n}$, взятых в произвольном порядке и с произвольной (но правильной) расстановкой скобок, запись $\left[\alpha_{1} \wedge \ldots \wedge \alpha_{n}\right]$ будет означать множество всех таких конъюнкций. Запись $\left[\alpha_{1} \wedge \ldots \wedge \alpha_{n} \rightarrow \alpha\right]$ 
будет обозначать множество всех импликаций, в посылках которых стоят конъюнкции, соответствующие данной квазиформуле. Пусть $X$ - некоторое конечное множество формул, посредством $X^{\wedge}$ будем обозначать некоторую конъюнкцию всех формул из этого множества.

Будем считать, что операция присоединения следствий $C$ в нашем языке уже задана и к тому же согласована с импликацией и конъюнкцией следующим образом:

B1. $\left[\alpha_{1} \wedge \ldots \wedge \alpha_{n} \rightarrow \alpha\right] \subseteq C(\varnothing) \Leftrightarrow \alpha \in C\left(\alpha_{1}, \ldots, \alpha_{n}\right)$.

Верно следующее утверждение:

TEOPЕМА 5. Условие В1 эквивалентно следующему множеству условий:

С1. C-стандартное следование;

C2. $\alpha \rightarrow \beta \in C(\varnothing) \Leftrightarrow \beta \in C(\alpha)$;

C3. $C(\alpha \wedge \beta)=C(\alpha, \beta)$.

Для его доказательства нам потребуется следующая

ЛЕММА 1. Пусть $C-$ такое следование, что для него верно, что $C(\alpha \wedge \beta)=C(\alpha, \beta)$, тогда для него верны равенства $C((\alpha \wedge$ $\beta) \wedge \gamma)=C(\alpha \wedge(\beta \wedge \gamma))=C(\alpha, \beta, \gamma)$.

\section{Доказательство.}

$C((\alpha \wedge \beta) \wedge \gamma)=C(\alpha \wedge \beta, \gamma)=C(C(\alpha \wedge \beta), C(\gamma))=$ $=C(C(\alpha, \beta), C(\gamma))=C(\alpha, \beta, \gamma)=C(C(\alpha), C(\beta, \gamma))=$ $=C(C(\alpha), C(\beta \wedge \gamma))=C(\alpha, \beta \wedge \gamma)=C(\alpha \wedge(\beta \wedge \gamma))$.

Q.E.D.

Доказательство. (Доказательство теоремы 5).

$(\Rightarrow)$ 1) Финитарность операции $C$ следует из того, что $\alpha \in C(X)$ тогда и только тогда, когда существует такое конечное множество формул $\left\{\alpha_{1}, \ldots, \alpha_{n}\right\} \subseteq X$, что $\left[\alpha_{1} \wedge \ldots \wedge \alpha_{n} \rightarrow \alpha\right] \subseteq$ $C(\varnothing)$.

Поскольку множество $C(\varnothing)$ замкнуто относительно всех подстановок, то для любой подстановки $\varepsilon$ верно, что если формула $\alpha_{1} \wedge \ldots \wedge \alpha_{n} \rightarrow \alpha \in C(\varnothing)$, то и $\varepsilon \alpha_{1} \wedge \ldots \wedge \varepsilon \alpha_{n} \rightarrow \varepsilon \alpha \in C(\varnothing)$. 
Таким образом, для любой формулы $\alpha$ и любой подстановки $\varepsilon$ верно, что если $\alpha \in C(X)$, то и $\varepsilon \alpha \in C(\varepsilon X)$. Откуда следует, что $\varepsilon C(X) \subseteq C(\varepsilon X)$.

2) Условие С2 совпадает с условием $\mathrm{B} 1$, при $n=1$.

3) По условию В1:

$$
\gamma \in C(\alpha, \beta) \Leftrightarrow[\alpha \wedge \beta \rightarrow \gamma] \subseteq C(\varnothing) \Leftrightarrow \gamma \in C(\alpha \wedge \beta) .
$$

$(\Leftarrow)$ Индукцией по $n \geq 1$ докажем, что из условий $\mathrm{C} 1-\mathrm{C} 3$ следует условие $B 1$.

Базис очевиден. Пусть для $k=n-1$ верно, что

$$
\alpha \in C\left(\alpha_{1}, \ldots, \alpha_{k}\right) \Leftrightarrow\left[\alpha_{1} \wedge \ldots \wedge \alpha_{k} \rightarrow \alpha\right] \subseteq C(\varnothing) .
$$

Таким образом, $C\left(\alpha_{1}, \ldots, \alpha_{n-1}\right)=C\left(\alpha_{1} \wedge \ldots \wedge \alpha_{n-1}\right)$.

Пусть $\alpha \in C\left(\alpha_{1}, \ldots, \alpha_{n}\right)$. Поскольку верно следующее включение $-C\left(\alpha_{1} \wedge \ldots \wedge \alpha_{n-1}\right) \subseteq C\left(\alpha_{1} \wedge \ldots \wedge \alpha_{n-1}, \alpha_{n}\right)$, то $\left\{\alpha_{1}, \ldots, \alpha_{n}\right\} \subseteq C\left(\alpha_{1} \wedge \ldots \wedge \alpha_{n-1}, \alpha_{n}\right)$. Следовательно, верно, что $C\left(\left\{\alpha_{1}, \ldots, \alpha_{n}\right\}\right) \subseteq C\left(C\left(\alpha_{1} \wedge \ldots \wedge \alpha_{n-1}, \alpha_{n}\right)\right)$. При этом в силу условия С3 и леммы 1 , мы получаем следующее равенство $C\left(\alpha_{1} \wedge \ldots \wedge \alpha_{n-1}, \alpha_{n}\right)=C\left(\alpha_{1} \wedge \ldots \wedge \alpha_{n-1} \wedge \alpha_{n}\right)$. Также имеем, что $C\left(C\left(\alpha_{1} \wedge \ldots \wedge \alpha_{n-1}, \alpha_{n}\right)\right)=C\left(\alpha_{1} \wedge \ldots \wedge \alpha_{n-1}, \alpha_{n}\right)$. Поэтому $C\left(\alpha_{1}, \ldots, \alpha_{n}\right) \subseteq C\left(\alpha_{1} \wedge \ldots \wedge \alpha_{n}\right)$. Таким образом, $\alpha \in C\left(\alpha_{1} \wedge \ldots \wedge \alpha_{n}\right)$ и, следовательно, в силу условия С2 верно, что $\left[\alpha_{1} \wedge \ldots \wedge \alpha_{n} \rightarrow \alpha\right] \subseteq C(\varnothing)$.

Пусть $\left[\alpha_{1} \wedge \ldots \wedge \alpha_{n} \rightarrow \alpha\right] \subseteq C(\varnothing)$ и, значит, $\alpha \in C\left(\alpha_{1} \wedge \ldots \wedge\right.$ $\left.\alpha_{n}\right)$. Так как $\left\{\alpha_{1}, \ldots, \alpha_{n-1}\right\} \subseteq\left\{\alpha_{1}, \ldots, \alpha_{n}\right\}$, то следовательно $C\left(\alpha_{1} \wedge \ldots \wedge \alpha_{n-1}\right) \subseteq C\left(\alpha_{1}, \ldots, \alpha_{n}\right)$. Таким образом, формула $\alpha_{1} \wedge \ldots \wedge \alpha_{n-1} \in C\left(\alpha_{1}, \ldots, \alpha_{n}\right)$. Отсюда не сложно заметить следующее включение: $\left\{\alpha_{1} \wedge \ldots \wedge \alpha_{n-1}, \alpha_{n}\right\} \subseteq C\left(\alpha_{1}, \ldots, \alpha_{n}\right)$. Следовательно, получаем, что $C\left(\alpha_{1} \wedge \ldots \wedge \alpha_{n}\right) \subseteq C\left(\alpha_{1}, \ldots, \alpha_{n}\right)$.

Q.E.D.

Логика, следование в которой удовлетворяет условиям C1-C3, в работах Ризарда Вуйцицкого ([1] и [2]) была названа хорошо определенной логикой (well-determined logic). Там же определено и понятие дедуктивного множества формул.

Далее следование в хорошо определенной логике мы будем называть хорошо определенным следованием. 


\section{5 Дедуктивные множества}

Множество формул $L$ будем называть дедуктивным тогда и только тогда, когда существует такая хорошо определенная логика $C$, что $C(\varnothing)=L$.

Формулы, содержащие в качестве связки только конъюнкцию, везде далее будем обозначать маленькими греческими буквами с индексом $\wedge$, например $\alpha^{\wedge}$. Множество пропозициональных переменных, входящих в формулу $\alpha$, будем обозначать $\operatorname{Var}(\alpha)$.

ЛЕММА 2. Если множество L является дедуктивным, то оно удовлетворяет следующим условиям:

1. Множество L замкнуто относительно всех подстановок.

2. Для любых формул $\alpha^{\wedge}$ и $\beta^{\wedge}$ верно, что если имеет место включение $\operatorname{Var}\left(\beta^{\wedge}\right) \subseteq \operatorname{Var}\left(\alpha^{\wedge}\right), \operatorname{mo~} \alpha^{\wedge} \rightarrow \beta^{\wedge} \in L$.

3. Множество L замкнуто относительно следующих правил вывода:

$$
\begin{array}{ccc}
(T R) \frac{p \rightarrow q, q \rightarrow r}{p \rightarrow r} & (C M) \frac{p_{1} \rightarrow q_{1}, p_{2} \rightarrow q_{2}}{p_{1} \wedge p_{2} \rightarrow q_{1} \wedge q_{2}} & (A D) \frac{p, q}{p \wedge q} \\
(C V) \frac{p, p \wedge q \rightarrow r}{q \rightarrow r} & (M P) \frac{p, p \rightarrow q}{q} & (E A) \frac{p \rightarrow q}{p \wedge r \rightarrow q}
\end{array}
$$

Доказательство. Пусть множество $L$ дедуктивно, значит, существует такое хорошо определенное стандартное следование $C$, что $C(\varnothing)=L$.

1. Поскольку $C$ - структурное, то множество $C(\varnothing)=L$ замкнуто относительно всех подстановок.

2. В силу леммы 1 и свойства С 3 верны следующие равенства: $C\left(\alpha^{\wedge}\right)=C\left(\operatorname{Var}\left(\alpha^{\wedge}\right)\right)$ и $C\left(\beta^{\wedge}\right)=C\left(\operatorname{Var}\left(\beta^{\wedge}\right)\right)$. Тогда, если $\operatorname{Var}\left(\beta^{\wedge}\right) \subseteq \operatorname{Var}\left(\alpha^{\wedge}\right)$, то, следовательно, $C\left(\operatorname{Var}\left(\beta^{\wedge}\right)\right) \subseteq C\left(\operatorname{Var}\left(\alpha^{\wedge}\right)\right)$ и значит $C\left(\beta^{\wedge}\right) \subseteq C\left(\alpha^{\wedge}\right)$. Таким образом, $\beta^{\wedge} \in C\left(\alpha^{\wedge}\right)$, откуда следует, что $\alpha^{\wedge} \rightarrow \beta^{\wedge} \in C(\varnothing)$.

3. (TR) Пусть $p \rightarrow q \in C(\varnothing)$ и $q \rightarrow r \in C(\varnothing)$. Значит $q \in C(p)$ и $r \in C(q)$. Так как по следствию $1 C(q) \subseteq C(p)$, то $r \in C(p)$ и, следовательно, $p \rightarrow r \in C(\varnothing)$. 
$(\mathrm{CM})$ Пусть $p_{1} \rightarrow q_{1} \in C(\varnothing)$ и $p_{2} \rightarrow q_{2} \in C(\varnothing)$, тогда $q_{1} \in$ $C\left(p_{1}\right)$ и $q_{2} \in C\left(p_{2}\right)$. Так как $\left\{p_{1}\right\} \subseteq\left\{p_{1}, p_{2}\right\},\left\{p_{2}\right\} \subseteq\left\{p_{1}, p_{2}\right\}$ и, по теореме $5, C\left(p_{1}, p_{2}\right)=C\left(p_{1} \wedge p_{2}\right)$, то верно, что $C\left(p_{1}\right) \subseteq C\left(p_{1} \wedge p_{2}\right)$ и $C\left(p_{2}\right) \subseteq C\left(p_{1} \wedge p_{2}\right)$. Таким образом, множество $\left\{q_{1}, q_{2}\right\} \subseteq C\left(p_{1} \wedge\right.$ $\left.p_{2}\right)$. Тогда, по теореме $2, C\left(q_{1}, q_{2}\right) \subseteq C\left(p_{1} \wedge p_{2}\right)$. Отсюда следует, что $C\left(q_{1} \wedge q_{2}\right) \subseteq C\left(p_{1} \wedge p_{2}\right)$, и, в силу указанного выше следствия, $q_{1} \wedge q_{2} \in C\left(p_{1} \wedge p_{2}\right)$. Следовательно, $p_{1} \wedge p_{2} \rightarrow q_{1} \wedge q_{2} \in C(\varnothing)$.

$(\mathrm{CV})$ Пусть $p \in C(\varnothing)$ и $p \wedge q \rightarrow r \in C(\varnothing)$. В силу свойства А 3 из первого следует, что $p \in C(q)$, и, значит, по теореме 1 , $C(p, q)=C(q)$. Из второго условия следует, что $r \in C(p, q)$. Таким образом, $r \in C(q)$ и, значит, $q \rightarrow r \in C(\varnothing)$.

(MP) Пусть $p \in C(\varnothing)$ и $p \rightarrow q \in C(\varnothing)$. Таким образом, $q \in$ $C(p)$ и $C(p) \subseteq C(\varnothing)$. Значит, $q \in C(\varnothing)$.

$(\mathrm{AD})$ Пусть $p \in C(\varnothing)$ и $q \in C(\varnothing)$. Тогда, по свойствам $\mathrm{A} 2$ и A3, получаем, что $C(p, q) \subseteq C(\varnothing)$, а, значит, и $C(p \wedge q) \subseteq C(\varnothing)$. Таким образом, $p \wedge q \in C(\varnothing)$.

(ЕА) Пусть $p \rightarrow q \in C(\varnothing)$. Таким образом, $q \in C(p)$. Так как $\{p\} \subseteq\{p, r\}$, то $C(p) \subseteq C(p, r)$ и, значит, $q \in C(p, r)$. Следовательно, $p \wedge r \rightarrow q \in C(\varnothing)$.

Q.E.D.

Пусть $L-$ непустое множество формул. Посредством $\vec{L}$ обозначим одноместную операцию на множестве $2^{S}$, котрую определим следующим образом:

(1) $\alpha \in \vec{L}(X) \Leftrightarrow \exists \alpha_{1}, \ldots, \alpha_{n} \in X \cup L\left(\alpha_{1} \wedge \ldots \wedge \alpha_{n} \rightarrow \alpha \in L\right)$.

Пусть $X$ - некоторое множество, посредством $R_{X}$ будем обозначать множество всех правил вывода, относительно которых множество $X$ замкнуто.

TEOPEMA 6. Пусть $L-$ некоторое множество формул, замкнутое относительно подстановки, для которого верно, что $\left\{\alpha^{\wedge} \rightarrow \beta^{\wedge} \mid \operatorname{Var}\left(\beta^{\wedge}\right) \subseteq \operatorname{Var}\left(\alpha^{\wedge}\right)\right\} \subseteq L u\{(T R),(C M)\} \subseteq R_{L}$. Toгда $\vec{L}$ является стандартным следованием, причем $L \subseteq \vec{L}(\varnothing)$.

Доказательство. Докажем, что $\vec{L}$ является операцией присоединения следствий.

(A1) Пусть $\alpha \in X$, так как $\alpha \rightarrow \alpha \in L$, то, по (1), $\alpha \in \vec{L}(X)$.

(А3) Пусть $X \subseteq Y$. Если $\alpha \in \vec{L}(X)$, то, по условию (1), $\exists \alpha_{1}, \ldots, \alpha_{n} \in X \cup L\left(\alpha_{1} \wedge \ldots \wedge \alpha_{n} \rightarrow \alpha \in L\right)$. Так как $X \subseteq Y$, то $\alpha_{1}, \ldots, \alpha_{n} \in Y \cup L$ и, значит, $\alpha \in \vec{L}(Y)$. 
(А2) Включение $\vec{L}(X) \subseteq \vec{L}(\vec{L}(X))$ следует из А1. Докажем обратное включение.

Пусть формула $\alpha \in \vec{L}(\vec{L}(X))$, тогда в силу условия (1) верно, что $\exists \alpha_{1}, \ldots, \alpha_{n} \in L \cup \vec{L}(X)\left(\alpha_{1} \wedge \ldots \wedge \alpha_{n} \rightarrow \alpha \in L\right)$.

Заметим, что если $\alpha \in L$, то, так как $\alpha \rightarrow \alpha \in L$, имеем $\alpha \in \vec{L}(\varnothing)$. Следовательно, $L \subseteq \vec{L}(\varnothing)$ и, значит, $L \cup \vec{L}(X)=\vec{L}(X)$.

Таким образом, для любого $1 \leq i \leq n$, верно, что $\alpha_{i} \in \vec{L}(X)$ и, значит, в силу (1), существуют формулы $\alpha_{i 1}, \ldots, \alpha_{i m_{i}} \in L \cup X$ такие, что $\alpha_{i 1} \wedge \ldots \wedge \alpha_{i m_{i}} \rightarrow \alpha_{i} \in L$.

Введем следующие обозначения. Положим $\beta_{i}=\alpha_{i 1} \wedge \ldots \wedge \alpha_{i m_{i}}$, $\beta^{1}=\beta_{1}, \alpha^{1}=\alpha_{1}$ и для любого $1 \leq k \leq n-1, \beta^{k+1}=\beta^{k} \wedge \beta_{k+1}$ и $\alpha^{k+1}=\alpha^{k} \wedge \alpha_{k+1}$.

Применяем (CM). Для любого $1 \leq k \leq n-1$ :

$$
\frac{\beta^{k} \rightarrow \alpha^{k}, \beta_{k+1} \rightarrow \alpha_{k+1}}{\beta^{k+1} \rightarrow \alpha^{k+1}} .
$$

Затем применяем (TR):

$$
\frac{\beta^{n} \rightarrow \alpha^{n}, \alpha^{n} \rightarrow \alpha}{\beta^{n} \rightarrow \alpha}
$$

Таким образом, формула $\beta^{n} \rightarrow \alpha \in L$. Поскольку при этом верно, что $\left\{\alpha_{11}, \ldots, \alpha_{1 m_{1}}, \ldots, \alpha_{n 1}, \ldots, \alpha_{n m_{n}}\right\} \subseteq X \cup L$, то $\alpha \in \vec{L}(X)$. Следовательно $\vec{L}(\vec{L}(X)) \subseteq \vec{L}(X)$ и, значит, $\vec{L}(X)=\vec{L}(\vec{L}(X))$.

Чтобы доказать стандартность следования $\vec{L}$, докажем сначала его структурность.

Пусть $\alpha \in \varepsilon \vec{L}(X)$, тогда существует такая формула $\beta$, что $\alpha=\varepsilon \beta$, и существуют формулы $\alpha_{1}, \ldots, \alpha_{n} \in L \cup X$ такие, что формула $\alpha_{1} \wedge \ldots \wedge \alpha_{n} \rightarrow \beta \in L$. Так как множество $L$ замкнуто относительно любой подстановки, то $\varepsilon \alpha_{1} \wedge \ldots \wedge \varepsilon \alpha_{n} \rightarrow \varepsilon \beta \in L$. Поскольку при этом $\varepsilon \alpha_{1}, \ldots, \varepsilon \alpha_{n} \in L \cup \varepsilon X$, то $\alpha=\varepsilon \beta \in \vec{L}(\varepsilon X)$. Таким образом, $\varepsilon \vec{L}(X) \subseteq \vec{L}(\varepsilon X)$.

Остается доказать финитарность. Пусть $\alpha \in \vec{L}(X)$, тогда, по условию (1), $\exists \alpha_{1}, \ldots, \alpha_{n} \in L \cup X\left(\alpha_{1} \wedge \ldots \wedge \alpha_{n} \rightarrow \alpha \in L\right)$. Пусть $Y=\left\{\alpha_{1}, \ldots, \alpha_{n}\right\} \cap X$. По условию (1) получаем, что $\alpha \in \vec{L}(Y)$, так как $\exists \alpha_{1}, \ldots, \alpha_{n} \in L \cup Y\left(\alpha_{1} \wedge \ldots \wedge \alpha_{n} \rightarrow \alpha \in L\right)$. Заметим, что множество $Y$ является конечным подмножеством множества $X$.

Q.E.D. 
Далее операцию $\vec{L}$, определенную для множества $L$, удовлетворяющего условиям теоремы 6 , будем называть импликативным следованием над множеством $L$.

ЛЕММА 3. Пусть L-некоторое множество формул, для которого выполненны условия теоремы 6. Для следования $\vec{L}$ верно, ито $\vec{L}(\varnothing)=L$, если $\{(A D),(M P)\} \subseteq R_{L}$.

Доказательство. Включение $L \subseteq \vec{L}(\varnothing)$ доказано в теореме 6 , докажем обратное включение. Пусть $\alpha \in \vec{L}(\varnothing)$, следовательно существуют формулы $\alpha_{1}, \ldots, \alpha_{n} \in L$ такие, что формула $\alpha_{1} \wedge$ $\ldots \wedge \alpha_{n} \rightarrow \alpha \in L$. Применяя $n-1$ раз правило (AD), получим, что $\alpha_{1} \wedge \ldots \wedge \alpha_{n} \in L$ и, следовательно, по правилу (МР), $\alpha \in L$.

Q.E.D.

TEOPEMA 7. Пусть L-некоторое множество формул. Oперация $\vec{L}$ является хорошо определенным следованием, для которого $\vec{L}(\varnothing)=L$, если и только если

$L$ замкнуто относительно подстановки,

$$
\begin{aligned}
& \left\{\alpha^{\wedge} \rightarrow \beta^{\wedge} \mid \operatorname{Var}\left(\beta^{\wedge}\right) \subseteq \operatorname{Var}\left(\alpha^{\wedge}\right)\right\} \subseteq L u \\
& \{(T R),(C M),(A D),(M P),(C V),(E A)\} \subseteq R_{L} .
\end{aligned}
$$

Доказательство. $(\Rightarrow)$ Следует из леммы 2.

$(\Leftarrow)$ Докажем, что $\vec{L}$ является следованием хорошо определенной логики.

(C1) Что $\vec{L}$ является стандартным следованием, для которого $\vec{L}(\varnothing)=L$, доказано в теореме 6 и лемме 3 .

$(\mathrm{C} 2)(\Leftarrow)$ Пусть $\alpha \in \vec{L}(\beta)$, тогда существуют такие формулы $\alpha_{1}, \ldots, \alpha_{n} \in L \cup\{\beta\}$, что $\alpha_{1} \wedge \ldots \wedge \alpha_{n} \rightarrow \alpha \in L$.

Возможны два случая.

1) Формула $\beta \notin\left\{\alpha_{1}, \ldots, \alpha_{n}\right\}$. Так как $\alpha_{1} \wedge \ldots \wedge \alpha_{n} \rightarrow \alpha \in L$, то, используя правило (ЕА), получаем, что $\alpha_{1} \wedge \ldots \wedge \alpha_{n} \wedge \beta \rightarrow \alpha \in L$.

2) Для некоторого $1 \leq i \leq n$ формула $\beta=\alpha_{i}$. Заметим, что поскольку $\left\{\alpha^{\wedge} \rightarrow \beta^{\wedge} \mid \operatorname{Var}\left(\beta^{\wedge}\right) \subseteq \operatorname{Var}\left(\alpha^{\wedge}\right)\right\} \subseteq L$ и множество $L$ замкнуто отросительно подстановки и правила $(T R)$, то если верно, что $\alpha_{1} \wedge \ldots \wedge \alpha_{n} \rightarrow \alpha \in L$, то верно и $\left[\alpha_{1} \wedge \ldots \wedge \alpha_{n} \rightarrow \alpha\right] \subseteq L$. Тогда получаем, что

$$
\left(\alpha _ { 1 } \wedge \left(\ldots \wedge \left(\alpha _ { i - 1 } \wedge \left(\alpha_{i+1} \wedge\left(\ldots \wedge\left(\alpha_{n} \wedge \beta\right) \ldots\right) \rightarrow \alpha \in L .\right.\right.\right.\right.
$$


Теперь применением в обоих случаях соответствующего числа раз правила $(\mathrm{CV})$ получим, что $\beta \rightarrow \alpha \in L$.

$(\Rightarrow)$ Пусть $\beta \rightarrow \alpha \in L$, тогда из определения операции $\vec{L}$ следует, что $\alpha \in \vec{L}(\beta)$.

$(\mathrm{C} 3)(\Leftarrow)$ Пусть формула $\alpha \in \vec{L}(\beta, \gamma)$, тогда существуют такие формулы $\alpha_{1}, \ldots, \alpha_{n} \in L \cup\{\beta, \gamma\}$, что $\alpha_{1} \wedge \ldots \wedge \alpha_{n} \rightarrow \alpha \in L$. Возможны следующие случаи.

1) Множество $\{\beta, \gamma\} \nsubseteq\left\{\alpha_{1}, \ldots, \alpha_{n}\right\}$. Значит, $\alpha_{1}, \ldots, \alpha_{n} \in L$ и тогда, применяя правила (AD) и (MP), получим, что $\alpha \in \vec{L}(\varnothing)$. Поскольку $\vec{L}(\varnothing) \subseteq \vec{L}(\beta \wedge \gamma)$, то $\alpha \in \vec{L}(\beta \wedge \gamma)$.

2) Для некоторого $1 \leq i \leq n$ формула $\beta=\alpha_{i}$ и $\gamma \notin\left\{\alpha_{1}, \ldots, \alpha_{n}\right\}$, тогда, как замечено для аналогичного случая выше, получаем, что $\left(\alpha_{1} \wedge\left(\ldots \wedge\left(\alpha_{i-1} \wedge\left(\alpha_{i+1} \wedge\left(\ldots \wedge\left(\alpha_{n} \wedge \beta\right) \ldots\right) \rightarrow \alpha \in L\right.\right.\right.\right.$. Применяя соответствующее число раз правило (CV), получим, что $\beta \rightarrow \alpha \in L$. Откуда, применяя правило (EA), получаем, что $\beta \wedge \gamma \rightarrow \alpha \in L$. Тогда, по определению, $\alpha \in \vec{L}(\beta \wedge \gamma)$.

3) Случай, когда для некоторого $1 \leq i \leq n$ формула $\gamma=\alpha_{i}$ и $\beta \notin\left\{\alpha_{1}, \ldots, \alpha_{n}\right\}$ доказывается аналогично.

4) Пусть $\{\beta, \gamma\} \subseteq\left\{\alpha_{1}, \ldots, \alpha_{n}\right\}$, как и выше, отсюда следет, что $\left(\alpha_{1} \wedge(\ldots \wedge(\beta \wedge \gamma) \ldots) \rightarrow \alpha \in L\right.$. Применяя соответствующее число раз правило $(\mathrm{CV})$, получим, что $\beta \wedge \gamma \rightarrow \alpha \in L$.

$(\Rightarrow)$ Пусть $\alpha \in \vec{L}(\beta \wedge \gamma)$, в силу доказанного выше пункта $\mathrm{C} 2$, получаем, что $\beta \wedge \gamma \rightarrow \alpha \in L$, и, значит, по определению операции $\vec{L}$, имеем, что $\alpha \in \vec{L}(\beta, \gamma)$.

Таким образом, $\vec{L}(\beta, \gamma)=\vec{L}(\beta \wedge \gamma)$.

Q.E.D.

Из леммы 2 и теоремы 7 непосредственно следует критерий дедуктивности множества формул.

TЕОРЕМА 8. Множество L является дедуктивным тогда и только тогда, когда оно удовлетворяет следующим условиям:

D1. Множество L замкнуто относительно всех подстановок.

D2. $\left\{\alpha^{\wedge} \rightarrow \beta^{\wedge} \mid \operatorname{Var}\left(\beta^{\wedge}\right) \subseteq \operatorname{Var}\left(\alpha^{\wedge}\right)\right\} \subseteq L$;

D3. $\{(T R),(C M),(A D),(M P),(C V),(E A)\} \subseteq R_{L}$.

Заметим, что таким образом минимальное дедуктивное множеcтво, которое мы будем обозначать буквой $W$, можно определить как множество всех формул, выводимых в исчислении со 
множеством тавтологий $\left\{\alpha^{\wedge} \rightarrow \beta^{\wedge} \mid \operatorname{Var}\left(\beta^{\wedge}\right) \subseteq \operatorname{Var}\left(\alpha^{\wedge}\right)\right\}$ и правилами вывода (TR), (CM), (AD), (MP), (CV) и (EA).

\section{6 Единственность хорошо определенного следования}

Введем следующие определения.

Пусть $C_{1}$ и $C_{2}$ - некоторые следования над одним и темже языком. Эти следования не совпадают, если существует такое множество $X$, что $C_{1}(X) \neq C_{2}(X)$. Будем говорить, что следование $C_{1}$ не сильнее следования $C_{2}$, если для любого множества $X$ верно, что $C_{1}(X) \subseteq C_{2}(X)$. Если имеет место тот факт, что $C_{1}$ не сильнее $C_{2}$ и $C_{1} \neq C_{2}$, то будем говорить, что $C_{1}$ слабее $C_{2}$ и $C_{2}$ сильнее $C_{1}$ и обозначать эти факты $C_{1}<C_{2}$ и $C_{2}>C_{1}$ соответственно.

Верна следующая

TEOPEMA 9. Пусть $L-$ - дедуктивное множество. Хорошо определенное следование $C$ такое, что $C(\varnothing)=L$ является единственным и совпадает с импликативным следованием $\vec{L}$ над множеством L.

Доказательство. Пусть $C$ - некоторое хорошо определенное следование такое, что $C(\varnothing)=L$. Докажем, что оно совпадает с импликативным следованием $\vec{L}$, откуда и будет следовать единственность такого следования.

Пусть $C \neq \vec{L}$, тогда либо $\vec{L}<C$, либо $C<\vec{L}$, либо $C$ несравнимо с $\vec{L}$.

Пусть $\vec{L}<C$, тогда существуют такое множество $X$, что $\vec{L}(X) \subset C(X)$, а значит, и такая формула $\alpha$, что $\alpha \in C(X)$ и $\alpha \notin \vec{L}(X)$. Поскольку $C$ - финитарное, то, следовательно, существует такое конечное множество $Y \subseteq X$, что $\alpha \in C(Y)$, причем $\alpha \notin \vec{L}(Y)$. Так как $C$ - хорошо опредленное следование, то $Y^{\wedge} \rightarrow \alpha \in C(\varnothing)=L$, тогда, по определению импликативного следования, $\alpha \in \vec{L}(Y)$. Противоречие.

Пусть $C<\vec{L}$, тогда существует такое множество $X$, что $C(X) \subset$ $\vec{L}(X)$, а значит, и такая формула $\alpha$, что $\alpha \notin C(X)$ и $\alpha \in \vec{L}(X)$. Из последнего следует, что существует конечное множество формул $Y=\left\{\alpha_{1}, \ldots, \alpha_{n}\right\} \subseteq X \cup L$, причем такое, что $\alpha_{1} \wedge \ldots \wedge \alpha_{n} \rightarrow$ $\alpha \in L$. Введем следующие обозначения. Посредством $Z_{L}$ обозна- 
чим конъюнкцию всех формул из множества $Y \cap L$. Положим $Z=Y \backslash L \subseteq X$. В силу теоремы 8 имеем, что $Z_{L} \wedge Z^{\wedge} \rightarrow \alpha \in L$ и $Z^{\wedge} \rightarrow \alpha \in L$. Так как $Z \subseteq X$ и $L=C(\varnothing)$, то $\alpha \in C(X)$. Противоречие.

Пусть $C$ и $\vec{L}$ несравнимы друг с другом, тогда существуют такие множества $X$ и $Y$ и такие формулы $\alpha$ и $\beta$, что $\alpha \in C(X)$ и $\alpha \notin \vec{L}(X)$, и $\beta \notin C(X)$ и $\beta \in \vec{L}(X)$. Далее доказательство аналогично доказательствам для случаев, приведенных выше.

Таким образом, $C=\vec{L}$.

Q.E.D.

\section{7 Хорошо определенные логики и теорема о дедукции}

Заметим, что тот факт, что $\alpha \in C(\beta)$, означает, что формулы $\alpha$ и $\beta$ находятся над данной логикой в отношении выводимости, что в более привычных обозначениях записывается как $\alpha \vdash \beta$. Тогда условие $(\mathrm{C} 2)$ теоремы 5 запишется знакомым образом

$$
\alpha \vdash \beta \Leftrightarrow \vdash \alpha \rightarrow \beta .
$$

Таким образом, условие $(\mathrm{C} 2)$ есть не что иное, как слабый вариант теоремы о дедукции. Это означает, что теорема о дедукции должна рассматриваться не только как технический момент, упрощающий построение выводов, но и как сообщение о некоторой согласованности языка и логики этого языка. А именно как сообщение о том, что язык обладает некоторыми средствами, позволяющими высказываться о логике этого же языка.

Если для логики верна теорема о дедукции в слабой ее форме, то будем говорить, что логика обладает слабым дедуктивным свойством или является слабо-дедуктивной.

Из теорем 8 и 9 следует

TЕОРЕМА 10. Минимальной логикой в языке, содержащем импликаиию и конгюнкиию, обладающей слабым дедуктивным свойством, является логика с импликативным следованием $\vec{W}$ над множеством $W$.

Минимальные логики с дедуктивным свойством исследовались В.А. Смирновым. В работе [3] им были представлены некоторые такие логики. Также, Х. Карри в книге [4] был приведен достаточный критерий для слабой дедуктивности некото- 
рых логик. Однако в указанных работах рассматривались логики, для теорий которых постулировалась замкнутость относительно правила modus ponens, кроме того, логики найденные В.А. Смирновым обладают и сильным дедуктивным свойством. Таким образом, результаты Р. Вуйцицкого, а также автора данной работы являются обобщением дедуктивного свойства логик в несколько ином направлении.

\section{Литература}

[1] Wojcicki R. Lectures on Propositional Calculi // www.studialogica.org/wojcicki

[2] Wojcicki R. Lectures on Propositional Calculi // Wroclaw: Ossolineum, 1984.

[3] Смирнов В.А. Формальный вывод и логические исчисления. М., 1972.

[4] Хаскел Б. Карри Основания математической логики. М., 1969. 